\title{
RECONFIGURACIÓN DE LOS DERECHOS FUNDAMENTALES DE LOS TRABAJADORES FRENTE AL USO DE LA INTELIGENCIA ARTIFICIAL*
}

RECONFIGURATION OF THE FUNDAMENTAL RIGHTS OF WORKERS AGAINST THE USE OF ARTIFICIAL INTELLIGENCE RECONFIGURATION DES DROITS FONDAMENTAUX DES TRAVAILLEURS CONTRE L'UTILISATION DE L'INTELLIGENCE ARTIFICIELLE

Jesús Aguilera Durán**

RESUMEN: El uso de la tecnología avanza con paso firme y avasallador sobre las formas de trabajo que tradicionalmente se han utilizado. Con el uso de la inteligencia artificial en la mano de obra, fuentes laborales importantes se ven amenazadas y aunque se resisten al cambio, se avizora un panorama nada alentador porque la tecnología va modificando los medios de producción y desvalorizando a la persona como eje fundamental de un proceso en el cual jugaba un rol preponderante. Esta disrupción ocasiona la deshumanización de la mano de obra y, por ende, atenta contra los derechos fundamentales de los trabajadores. Para mitigar los efectos que este fenómeno está ocasionando, se requiere una reconfiguración normativa sobre los derechos laborales que propicie la convivencia entre personas y robots, pero conservando la primacía de lo humano sobre lo artificial.

Palabras clave: derechos fundamentales, mano de obra, inteligencia artificial.

* Recibido el 5 de mayo de 2020 y aceptado para su publicación el 3 de septiembre de 2020 .

** Profesor de Derechos humanos y Derecho constitucional en la Facultad de Derecho y Ciencias Sociales de la Universidad Autónoma del Estado de Morelos. Correo electrónico: jad-2009@live.com.mx.

T) Revista Latinoamericana de Derecho Social Núm. 31, julio-diciembre de 2020, pp. 51-70 
ABSTRACT: The use of technology advances with a firm and overwhelming step over the forms of work that have traditionally been used. With the use of artificial intelligence in the workforce, important labor sources are threatened and although they resist change, an unencouraging outlook is seen because technology is modifying the means of production and devaluing the person as the fundamental axis of a process in which he played a preponderant role. This disruption causes the dehumanization of the workforce and therefore, undermines the fundamental rights of workers. To mitigate the effects that this phenomenon is causing, it requires a normative reconfiguration on labor rights that encourages coexistence between people and robots while preserving the primacy of the human over the artificial.

Keywords: Fundamental Rights, Workforce, Artificial Intelligence.

RÉSUMÉ: L'utilisation de la technologie progresse avec une étape ferme et écrasante sur les formes de travail traditionnellement utilisées. Avec l'utilisation de l'intelligence artificielle dans la main-d'œuvre, d'importantes sources de main-d'œuvre sont menacées et bien qu'elles résistent au changement, un panorama peu encourageant est envisagé car la technologie modifie les moyens de production et dévalorise la personne comme axe fondamental de un processus dans lequel il a joué un rôle prépondérant. Cette perturbation entraîne la déshumanisation de la main-d'œuvre et, par conséquent, porte atteinte aux droits fondamentaux des travailleurs. Pour atténuer les effets de ce phénomène, une reconfiguration normative des droits du travail qui encourage la coexistence entre les personnes et les robots est nécessaire mais en préservant la primauté de l'humain sur l'artificiel.

Mots-clés: droits fondamentaux, travail, intelligence artificielle. 
SUMARIO: I. Introducción. II. Derechos fundamentales de los trabajadores. III. Inteligencia artificial en la mano de obra. IV. Expectativas sobre el futuro del trabajo. V. Reconfiguración de los derechos fundamentales de los trabajadores. VI. Conclusiones. VII. Fuentes de información.

\section{INTRODUCCIÓN}

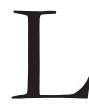

os derechos fundamentales de los trabajadores se ven afectados por los constantes cambios que suceden tanto en México como en otras regiones del mundo. Se dice que la causa más directa es el fenómeno de la globalización, aunque se debe ser cauteloso al afirmar una postura en este sentido, porque más bien pudiera ser una consecuencia de la expansión de la tecnología.

Esto, propiciado por los medios de comunicación, permitió, en términos de Giddens, que hubiera una dislocación de tiempo y espacio. ${ }^{1}$ Con ello, se conocieron nuevas formas de trabajar, se tuvo acceso a nuevas tecnologías y se fueron desvaneciendo los derechos de los trabajadores que son producto de una lucha histórica plagada de abusos y discriminación.

Sin darle demasiada importancia al uso tan invasivo de la tecnología en los medios de producción, los trabajadores parece que no vislumbran los alcances que el uso de la inteligencia artificial en la mano de obra está ocasionando. En otras palabras, se está deshumanizando el proceso productivo para mecanizarlo, con el consecuente impacto en el empleo.

Por lo anterior, se hace necesaria una reconfiguración de los derechos fundamentales de los trabajadores, que permita, tanto la protección y la garantía de dichos derechos ante la posible pérdida de empleos, como la capacitación laboral en prospectiva, para que cuando se agoten algunos empleos, los trabajadores perjudicados tengan una pronta reconexión con otra fuente laboral.

Metodológicamente se hace uso de la estrategia de sistematización de información sobre los derechos fundamentales de los trabajadores y el uso de la inteligencia artificial que va invadiendo espacios laborales en campos cada vez más diversos con el objeto de revisar la erosión que este hecho produce en los derechos y las expectativas laborales de las personas.

Para tal efecto, se recurre a fuentes de información secundarias con el auxilio de la técnica documental bibliográfica y la documental digital, para

1 Giddens, Anthony, As consequencias da modernidade, Sao Paulo, Editora UNESP, 1991, pp. 69 y 70 . 
recopilar información actualizada que permita realizar una interpretación del objeto de estudio con la finalidad de emitir conclusiones que describan el presente, pero más importante aún, que provoque una reflexión sobre cómo prepararse para seguir empleados en el futuro.

\section{DeREChOS Fundamentales DE los trabajADORES}

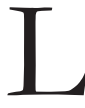

os derechos laborales han tenido que pasar por una larga defensa ante el poder del capital; esta lucha incesante se ha visto recompensada con logros en el reconocimiento de derechos tanto individuales como colectivos. La Constitución Política de los Estados Unidos Mexicanos de 1917 (en adelante CPEUM) fue pionera en el reconocimiento de los derechos sociales.

En el ámbito internacional, los tratados y las convenciones, principalmente sobre derechos humanos, han sido el eje sobre el que ha girado la búsqueda de mayores beneficios para los trabajadores.

Así pues, en la CPEUM, los derechos fundamentales de los trabajadores se protegen, principalmente, en los artículos 1o., 4o., 9o. y 123, y por virtud de los artículos 1o. y 133, también se reconoce su protección en los tratados internacionales en materia de derechos humanos.

En el artículo 1o. constitucional se establece la vinculación para todas las autoridades de "promover, respetar, proteger y garantizar los derechos humanos, los derechos laborales entre ellos de conformidad con los principios de universalidad, interdependencia, indivisibilidad y progresividad". ${ }^{2}$

Por lo que hace al artículo 4o., en éste se protege la organización y desarrollo de la familia, el derecho a la alimentación, a la salud, al desarrollo, al agua y a la vivienda, ${ }^{3}$ que son tan indispensables para garantizar una vida digna.

En el artículo 9o. se protege el derecho de asociarse pacíficamente y con un objeto licito, ${ }^{4}$ el cual ha sido utilizado para exigir, incluso por vía jurisdiccional, el derecho de formar sindicatos, aun en contra de las restricciones de las leyes secundarias en la materia.

Con respecto al artículo 123, establece que: “Toda persona tiene derecho al trabajo digno y socialmente útil; al efecto, se promoverán la creación de em-

2 Constitución Política de los Estados Unidos Mexicanos (CPEUM) de 5 de febrero de 1917, Diario Oficial de la Federación del 27 de agosto de 2018, p. 2, disponible en: http:/ / wmw. diputados.gob.mx/LeyesBiblio/pdf/1_270818.pdf.

3 Ibidem, pp. 7 y 8.

4 Ibidem, p. 13. 
pleos y la organización social de trabajo, conforme a la ley”. En el apartado A se regulan todas las relaciones laborales entre los trabajadores y los patrones, que pertenecen al sector privado y, excepcionalmente, a algunas entidades públicas. En el apartado B se regulan las relaciones de los poderes federales con sus trabajadores. ${ }^{5}$

En este precepto se regulan los contratos de trabajo, el salario mínimo, la jornada laboral, los días de descanso, la seguridad social, el pago de utilidades, los trabajos de menores y mujeres embarazadas, el acceso a la vivienda, la capacitación laboral, la sindicalización y la solución de conflictos,

Si bien es cierto que se le considera un catálogo enfocado a las relaciones laborales, también lo es que hay diversas situaciones que no se protegen ni garantizan, como las referentes a todas las nuevas actividades laborales que se originan por el uso y aplicación de la tecnología.

Sobre los tratados internacionales, es relevante recordar que el artículo 10. constitucional también incluye derechos humanos garantizados en diversos instrumentos internacionales como el Pacto Internacional de Derechos Económicos, Sociales y Culturales y su Protocolo Facultativo, la Organización Internacional del Trabajo, el Protocolo Adicional a la Convención Americana en Materia de Derechos Económicos, Sociales y Culturales, entre otros.

Es precisamente en los acuerdos internacionales donde se han protegido y garantizado los derechos humanos, entre ellos, el derecho [al trabajo], y nuestro país, al ser Estado parte en varios instrumentos internacionales, adquiere obligaciones para con los demás Estados y para con sus nacionales, tanto para impulsar reformas legislativas que reflejen lo convenido, como para impulsar políticas antidiscriminatorias. ${ }^{6}$

Así pues, en el Pacto Internacional de Derechos Económicos, Sociales y Culturales ${ }^{7}$ se protegen los derechos laborales, en el artículo 6o. el derecho a trabajar; en el artículo 7o. el derecho de toda persona al goce de condiciones de trabajo equitativas y satisfactorias; en el artículo 8o. el derecho de sindica-

5 Ibidem, pp. 127-138.

6 Aguilera Durán, Jesús, "Derecho de asociación de los trabajadores de confianza del municipio en el Estado de Morelos", en Cabrera Dircio, Julio et al., Las reformas constitucionales y su impacto en el municipio, México, Editorial Fontamara, 2015, pp. 167 y 168.

7 Pacto Internacional de Derechos Económicos, Sociales y Culturales, en línea, 16 de diciembre de 1966, entró en vigor el 3 de enero de 1976, disponible en: https:/ / wmw.ohchr.org/ sp/professionalinterest/pages/cescr.aspx. 
lización, en el artículo 9o. el derecho a la seguridad social; en el artículo 10 el trabajo de mujeres embarazadas y de menores; en el artículo 11 el derecho de toda persona a un nivel de vida adecuado para sí y su familia y en el artículo 12 el derecho a la salud y a la higiene en el trabajo.

Aunque el hecho de que se firmen no siempre va a representar un beneficio inmediato para los trabajadores, se pueden mencionar dos ejemplos al respecto, el primero con relación al Protocolo Facultativo del Pacto Internacional de Derechos Económicos, Sociales y Culturales adoptado el 10 de diciembre de 2008, el cual México no ha ratificado.

Se destaca que "el PF-PIDESC no crea ningún derecho sustantivo nuevo, sino un mecanismo que permite que personas o grupos presenten quejas ante el Comité de Derechos Económicos, Sociales y Culturales de Naciones Unidas, respecto de violaciones de estos derechos cometidas por un Estado parte". ${ }^{8}$ Pero precisamente por eso el Estado mexicano no lo quiere ratificar.

El segundo ejemplo lo es el Convenio 98 sobre el derecho de sindicación y de negociación colectiva de la Organización Internacional del Trabajo (OIT), el cual fue adoptado el 1o. de julio de 1949 y entró en vigor el 18 de julio de 1951, pero México lo ratificó en el Senado hasta el 20 de septiembre de 2018.

Tras casi tres años de estar en la "congeladora", el Senado aprobó por 78 votos a favor, cero en contra y ninguna abstención, el Convenio 98 de la Conferencia Internacional del Trabajo, sobre la aplicación de los principios del derecho de sindicatos y negociación colectiva, que implicará una reforma a la Ley Federal del Trabajo y la eliminación de los "contratos de protección", como los outsourcings. ${ }^{9}$

Como se puede observar, el Estado mexicano no garantiza los derechos laborales, aunque sean considerados derechos fundamentales, porque no es congruente con el cumplimiento de las obligaciones que le son impuestas por virtud de dichos instrumentos jurídicos del orden internacional.

Lo antes expuesto nos provoca la siguiente reflexión: si los derechos laborales fueron instituidos hace más de cincuenta años y apenas se hace algo por

8 OACNUDH, Carta del PF-PIDESC, disponible en: http://acnudh.org/wpcontent/ uploads/2010/12/ carta-PF-PIDESC-FINAL.pdf (fecha de consulta: 10 de diciembre de 2019).

9 Villamil, Jenaro, "El Senado ratifica el Convenio 98 sobre derechos sindicales y contra contratos de protección patronal”, Revista Proceso, en línea, núm. 2190, 20 de octubre de 2018, disponible en: https:// wmw.proceso.com.mx/551845/el-senado-ratifica-el-convenio-98-sobre-derechos-sindicales-y-contra-contratos-de-proteccion-patronal (fecha de consulta: 24 de diciembre de 2019). 
garantizarlos, ¿qué podemos esperar de la regulación de los derechos fundamentales de los trabajadores ante el embate del uso de la inteligencia artificial en la mano de obra?

\title{
III. INTELIGENCIA ARTIFICIAL EN LA MANO DE OBRA
}

\begin{abstract}
A analizar el uso de la inteligencia artificial en la actividad laboral, se 1 debe tener presente que la tecnología ha servido para mejorar muchas actividades las cuales tradicionalmente eran realizadas por una o varias personas.
\end{abstract}

Asimismo, la tecnología está cambiando al mundo, a la economía, a la sociedad, y a la cultura, y un sector social que está siendo especialmente sensible a estos cambios es el de las relaciones laborales. Siendo éste un sector siempre sujeto a transformaciones y evoluciones aceleradas, en los últimos años se detecta una intensificación de este fenómeno, consecuencia, entre otros factores, de los cambios tecnológicos experimentados... ${ }^{10}$

Lo anterior se puede observar desde dos aspectos, uno positivo y el otro negativo, en cuanto al primero, no se discute que todo cambio implica beneficios que, en este caso, van a ser más palpables para un sector del proceso productivo: los empresarios. Sin embargo, al optimizarse el intercambio de bienes y servicios, se afirma que también representa beneficios para los trabajadores,

... porque varios siglos de innovación han demostrado que, aun cuando las máquinas han podido hacer cada vez más tareas que los humanos solían hacer, esto lleva a los humanos a tener mayores ingresos, consumir más y crear empleos para casi todos los que los desean. En otras palabras, cuando los trabajadores han sido históricamente desplazados por la innovación tecnológica, se han trasladado a nuevos puestos de trabajo, que a menudo requieren tareas más complejas o niveles mayores de juicio independiente. ${ }^{11}$

10 Carrasco Fernández, Felipe Miguel, "Rescisión laboral por uso de medios electrónicos en la empresa", en Ibarra Sánchez, Ernesto y Romero Flores, Rodolfo, Jurismática. El derecho y las nuevas tecnologias, UANL, 2010, p. 173.

11 U.S. White House, Economic Report of the President 2016, en línea, disponible en: 
Se puede o no estar de acuerdo con esta postura, pero lo que es innegable es que el ser humano está dotado de raciocinio que le facilita desarrollar habilidades, aptitudes, destrezas y capacidades que le permiten reenfocarse en los requerimientos que la innovación tecnológica le exige para adaptarse y poder así acceder a mejores condiciones de trabajo.

En el aspecto negativo de dicha innovación, se considera que, a mediano o largo plazo, se va a presentar la disminución de empleos y la creación de otros nuevos, va a ser notorio el desfasamiento de los trabajadores en la capacitación digital, habrá incidencia de salarios no bien remunerados y ello podría ocasionar el desvanecimiento de los derechos fundamentales que gozan los trabajadores.

Con el tiempo, los economistas esperan que los salarios se ajusten para despejar el mercado laboral y los trabajadores respondan a los incentivos para desarrollar el capital humano. La desigualdad podría aumentar, de hecho, la mayoría de los economistas creen que el cambio tecnológico es parcialmente responsable del aumento de la desigualdad en las últimas décadas. ${ }^{12}$

Se supone que, al requerirse una mayor competitividad en la actividad laboral, se propicia la desigualdad, principalmente entre las nuevas generaciones, que se han formado en la era digital y aquellos trabajadores tradicionales que realizan trabajos sin requerimiento de cualificación alguna y que no han recibido el apoyo suficiente para capacitarse en el uso de la tecnología y, por lo tanto, no están preparados para enfrentar esos cambios.

"La automatización está presente desde hace tiempo en el sector industrial, donde cada vez más se hace uso de robots en el ámbito de los procesos productivos, en el ensamble y la manipulación de materiales, en las cadenas de montaje, en la confección de mercancías". ${ }^{13}$ Con ello, se intensifica la productividad, se reducen los costos y se generan más ganancias.

https:// obamawhitehouse.archives.gov/sites/default/files/docs/ERP_2016_Chapter_5.pdfp. 236 (fecha de consulta: 27 de diciembre de 2019).

12 Ibidem, p. 237.

13 Palmerini, Erika, "Robótica y derecho: sugerencias, confluencias, evoluciones en el marco de una investigación europea", Revista de Derecho Privado, en línea, núm. 32, enerojunio de 2017, p. 54, disponible en: https://revistas.uexternado.edu.co/index.php/derpri/article/ view/5021/6009 (fecha de consulta: 3 de enero de 2020). 
Esto sin dejar de ser positivo, provoca que se redimensione el mercado laboral, se produzca un impacto en los derechos laborales, se propicien formas de contratación temporales y se garanticen menos prestaciones sociales.

Sin ser una contradicción, la inteligencia artificial implica beneficios para unos, pero perjuicios para otros. "En la nueva ola de AI [Artificial Intelligence] existen oportunidades y retos al mismo tiempo. En el lado positivo, AI pudiera incrementar automatización, soporte de análisis inteligente y toma de decisiones, y crear nuevos modelos de negocios e industrias. Pero AI también trae una serie de riesgos". ${ }^{14}$

Esos riesgos, incluidos el desplazamiento laboral, la adaptación a nuevas formas de trabajo, el desvanecimiento de los derechos laborales, entre otros, se deben visualizar como un conflicto que en su momento deberán atender, tanto las autoridades, como los empresarios y, sin duda alguna, los trabajadores para que esa transición produzca los menos efectos posibles y se prevenga que las personas sufran, o al menos no resientan de forma brusca, en su economía personal, los efectos de la automatización.

\section{EXPECTATIVAS SOBRE EL FUTURO DEL TRABAJO}

\section{I}

os cambios tecnológicos van modificando la cadena de producción, por ende, la forma de trabajar, como lo describen David He y Vanessa Guo: "El impacto transformador de la inteligencia artificial (IA) en cada industria es indiscutible. Como lo es su efecto en el mercado laboral. ¿Beneficiará la IA al mercado laboral en la próxima década y más adelante, o lo cambiará y remplazará a los humanos?”.15

Esa pregunta invita a la reflexión, porque pareciera que las personas se preocupan más por estar atentos a actividades que en nada les beneficia como cuestiones políticas, sucesos naturales o simplemente en el celular perdiendo el tiempo, sin darse cuenta que el uso de la inteligencia artificial en la mano de obra va ocasionando el desplazamiento del trabajador de tareas en las que tradicionalmente eran su fortaleza y orgullo.

14 He, David y Vanessa, Guo, 4 ways AI [Artificial Intelligence] will impact the financial job market, World Economic Forum, traducción propia, disponible en: https://wmw.weforum.org/ agenda/2018/09/4-ways-ai-artificial-intelligence-impact-financial-job-market/ (fecha de consulta: 4 de enero de 2020).

15 Idem. 
Estos son las ocupaciones con las mayores posibilidades (99\% de probabilidad) de ser remplazadas por tecnología: ingreso de datos, bibliotecarios, nuevos contadores, trabajadores de procesos fotográficos, operadores de máquinas de procesamiento, preparadores de impuestos, agentes de carga y fletes, reparadores de relojes, agentes de seguros, técnicos matemáticos, alcantarillado, examinadores de títulos, abstractores, buscadores y telemarketers. ${ }^{16}$

Ante ese escenario, el futuro del trabajo se debe visualizar con responsabilidad, para poder enfrentar ese cambio allegados de las herramientas necesarias para una mejor adaptación a las nuevas actividades laborales, por eso, se debe capacitar permanentemente y desarrollar otras cualificaciones que permitan ser competitivos al solicitar un empleo.

Según un estudio del Centro Europeo para el Desarrollo de la Formación Profesional (CEDEFOP), de 2013 a 2025 se producirá un crecimiento en la demanda de perfiles comerciales y trabajadores de servicios, hasta ocupar un $27 \%$ de la demanda total de empleo en España, frente al 16\% de media en el resto de la Unión Europea. ${ }^{17}$

Entendiéndose por perfiles comerciales y de servicios aquellas personas altamente cualificadas con conocimientos digitales o informáticos para ocupar empleos en el sector de intercambio de bienes y servicios, que no sólo requieren actividades físicas sino más bien aptitudes intelectuales.

Eso llevará a que "... la labor de los departamentos de Recursos Humanos se basará en valorar el talento de una persona, así como sus habilidades y capacidades. Por tanto, cada negociación será única y estará vinculada directamente al perfil de cada trabajador de forma individual y no por colectivos". ${ }^{18}$

Es decir, el estar cualificado para desempeñar un puesto de trabajo, no tan sólo habrá de ser factor decisivo, sino que también se deben tener conocimientos sobre los derechos laborales porque al contratarse se determinarán

16 Dachs, Bernhard, The Impact of New Technologies on the Labour Market and the Social Economy, European Parliament, 2018, p. 25.

17 Repositorio institucional de la Fundación Universitaria San Pablo CEU, Informe ADDECO sobre el futuro del trabajo en España 2016, p. 52, disponible en: https: / wmw.ceu.es/joblab/ documentacion/informeAdecco.pdf (fecha de consulta: 10 de enero de 2020).

18 Ibidem, p. 130. 
las condiciones de trabajo que hoy se realizan a través de negociaciones colectivas.

"La evolución del mercado laboral y los cambios en las condiciones laborales y en las reivindicaciones de los empleados está dando lugar a la individualización. Los sindicatos han perdido poder de acción y de negociación a favor de los propios empleados". ${ }^{19}$ Esto puede ser ocasionado porque el actuar de las representaciones sindicales está más vinculado a intereses políticos, de grupo o particulares que a la defensa y mejora de los derechos del trabajador.

Indudablemente que se está dando una transición marcada por los diversos factores de la economía, lo que trae como consecuencia que se presenten problemas que hoy en día no son tan notorios, pero que se irán agudizando conforme la automatización vaya expandiéndose.

A pesar de lo expuesto, sí pueden perfilarse algunos de los riesgos que entraña, sea cual sea su intensidad y rapidez, la transición económica y laboral, como son el aumento temporal o más permanente del desempleo o la polarización del mercado de trabajo, abierto a una mayor brecha salarial y a la diferenciación de las condiciones laborales (en detrimento de quienes mantengan su trabajo en sectores y puestos de trabajo menos cualificados conforme a los parámetros de los cambios económicos). ${ }^{20}$

Con esto, no quiere decir que la mayoría de los empleos tiendan a desaparecer, sino que algunos se mudarán, otros se modificarán y muchos más se crearán, ello desde luego, se puede asimilar fortaleciendo una buena preparación académica, complementada con el desarrollo de conocimientos digitales a través de cursos, incluso on-line, que algunas veces, hasta pueden ser gratuitos. Esto se espera que suceda en la mayoría de los países.

Estimamos que para 2027, el 23\% de los empleos en el sector financiero de China serán recortados por AI [nteligencia Artificial] o se transformarán en nuevas posiciones. El 77\% restante de los trabajos no será remplazado, pero la eficiencia de estas posiciones aumentará. Los trabajos que serán

19 Ibidem, p. 129.

20 Bonet Pérez, Jordi, "Disrupción tecnológica y trabajo, ¿disrupción también en el ámbito de las relaciones laborales?”, Revista Mientras tanto.e, en línea, núm. 169, junio de 2018, p. 2, disponible en: http:// wmw.mientrastanto.org/boletin-169/notas/disrupcion-tecnologica-y-trabajo-disrupciontambien-en-el-ambito-de-las-relaciones (fecha de consulta: 11 de enero de 2020). 
remplazados por AI consisten principalmente en tareas estandarizadas y repetitivas. ${ }^{21}$

Eso nos brinda una idea de lo que debemos esperar en un futuro no muy lejano, hay que recordar que China es una de las economías más importantes del mundo, y en términos de mano de obra, un mercado laboral muy importante. Pero la automatización no afecta tan sólo a este país, sino prácticamente a todos los países industrializados o las economías emergentes más desarrolladas.

A nivel mundial, las actividades automatizables equivalen a 1.1 miles de millones de empleados y $\$ 15.8$ billones en salarios... Cuatro economías (China, India, Japón y los EE. UU.) representan un poco más de la mitad de estos salarios y empleados; China e India en su conjunto representan el mayor potencial de empleos automatizables (más de 700 millones de empleados equivalentes a tiempo completo) debido al tamaño relativo de sus fuerzas laborales. ${ }^{22}$

Lo antes expuesto no significa que los empleos desaparezcan, sino que van a sufrir variaciones tanto en la oferta como en la demanda, lo que implica una necesaria concientización de las expectativas que se deben tener para poder adaptarse a las necesidades futuras.

El potencial también es alto en Europa: de acuerdo con nuestro análisis, 54 millones de empleados equivalentes a tiempo completo o $\$ 1.7$ billones en salarios, están asociados con actividades automatizables en las 5 economías más importantes: Francia, Alemania, Italia, España y el Reino Unido...2.3

Con estos datos, que reflejan la trascendencia del fenómeno en los países industrializados, se impone hacer una valoración sobre el futuro del trabajo

21 He, David y Vanessa, Guo, op. cit.

22 Mckinzey Global Institute, "Un futuro que funciona: automatización, empleo y productividad”, Resumen ejecutivo 2017, p. 27, en línea, disponible en: https:// wmw.mckinsey.com/ / media/ mckinsey/featured\%20insights/digital\%20disruption/ harnessing\%20automation\%20for $\% 20$ a\%20 future\%20that\%20works/ a-future-that-works-executive-summary-spanish-mgi-march-24-2017.ashx' (fecha de consulta: 14 de enero de 2020).

23 Ibidem, p. 27. 
y el impacto que el uso de la inteligencia artificial va a tener en los derechos fundamentales de los trabajadores.

Esto sin contar que: "Las perspectivas de la FIR [Federación Internacional de Robótica] muestran que en 2021 el número anual de robots suministrados a fábricas alrededor del mundo alcanzará cerca de 630,000 unidades". ${ }^{24}$ Lo que visibiliza aún más la insoslayable interacción entre el hombre y los robots.

... 85 unidades de robot por cada 10,000 empleados es el nuevo promedio de densidad de robot global en las industrias manufactureras (2016: 74 unidades). Por regiones, la densidad promedio de robots en Europa es de 106 unidades, en América 91 y en Asia 75 unidades... 25

Ello implica que el futuro del trabajo está ligado a la robótica, y obliga a que se reconfiguren los derechos fundamentales de los trabajadores. Porque "es necesario un nuevo contrato social para una realidad que emana de la disrupción digital, tecnológica y científica. Avanzamos hacia una singularidad determinada por la confluencia de progresos vertiginosos que afectan a todos los órdenes de nuestra vida. ${ }^{26}$

\title{
V. RECONFIGURACIÓN DE LOS DERECHOS FUNDAMENTALES
}

DE LOS TRABAJADORES

\begin{abstract}
A nte la invasión de la tecnología en la mayor fuente de ingresos que tienen las personas: su fuerza de trabajo, y la erosión de la estabilidad en el empleo, se hace necesario que el Estado asuma con responsabilidad esos retos y que facilite a los trabajadores la adaptación a nuevas formas de trabajo, lo que a su vez traerá como consecuencia que se reconfiguren los derechos fundamentales de los trabajadores que, si llegaran a ser desplazados, estén preparados a iniciar actividades en otras áreas distintas a las que habían desempeñado.
\end{abstract}

24 IFR, Global Industrial Robot Sales Doubled over the Past Five Years, 18 de octubre de 2018, en línea, disponible en: https://ifr.org/ifr-press-releases/news/global-industrial-robot-sales-doubled-over-thepast-five-years (fecha de consulta: 24 de enero de 2020).

25 Idem.

26 Zafra, Juan Manuel, "Más humano y digital”, TELOS Revista de Pensamiento, Sociedad y Tecnología, núm. 108, enero-junio de 2018, p. 6. 
La perspectiva de que la tecnología disruptiva extienda aún más estas prácticas y abarque relativamente a un mayor número de integrantes (humanos) de la fuerza laboral, puede concitar la idea de una necesidad o aparente necesidad de cambio legal, con el argumento de que la nueva realidad económica y laboral derivada de la automatización y la digitalización exige una respuesta adecuada, ya que es un fenómeno inevitable que no hay más remedio que regular... ${ }^{27}$

Sin duda, que debe estar regulada la aplicación de nuevas tecnologías a las actividades laborales porque lleva implícita la interacción entre humanos y robots. Pero,

Para encontrar regulaciones legales sobre robótica e inteligencia artificial debemos irnos hasta Japón y Corea; Taiwán está investigando para establecer las bases de una futura normativa. Aparte de éstas, las únicas normas relativas a la robótica que hay en la actualidad son las normativas ISO (International Organization for Standardization). ${ }^{28}$

Estas normas ISO de carácter industrial, que son parte del soft law, "son documentos que especifican requerimientos que pueden ser empleados en organizaciones para garantizar que los productos y/o servicios ofrecidos por dichas organizaciones cumplen con su objetivo". ${ }^{29}$

Así pues, en Asia es donde se puede encontrar una mayor regulación sobre el uso de la robótica y la inteligencia artificial.

Se trata de la "Korean Law on the Development and Distribution of Intelligent Robots (2005)" y de la "Legal Regulation of Autonomous Systems in South Korea (2012)". En Japón podemos encontrar las "Guidelines to Secure the Safe Performance of Next Generation Robots" y la "New Robot Strategy - Japan's Robot Strategy - Vision, Strategy, Action Plan” y "Headquarters for Japan’s Economic Revitalization” de febrero (2015)...

27 Bonet Pérez, Jordi, op. cit., p. 3.

28 Pascual, Álvaro David, Inteligencia artificial: un panorama de algunos de sus desafíos éticos y jurídicos, Universitat de Girona, p. 35, en línea, disponible en: bttps://dugidoc.udg.edu/bitstream/ handle /10256/14950/alvaro-pascual.pdf? sequence=1 (fecha de consulta: 10 de febrero de 2020).

29 ISO Tools, “¿Qué es una norma ISO?”, en línea, disponible en: https:/ / wmw.isotools.org/ normas/ (fecha de consulta: 11 de febrero de 2020). 
En Europa, no se tiene como tal una regulación, pero el Parlamento Europeo aprobó el 16 de febrero de 2017 el texto P8_TA (2017)0051 que contiene recomendaciones destinadas a la Comisión sobre normas de derecho civil sobre robótica, el cual en su inciso L, señala:

Considerando que no cabe duda de que el desarrollo de la robótica y la inteligencia artificial incidirán en el panorama del empleo, lo que puede crear nuevas preocupaciones relativas a la responsabilidad y eliminar otras; que debe aclararse la responsabilidad jurídica desde el punto de vista del modelo de empresa y de la definición de las tareas de los trabajadores, en caso de que se produzca una emergencia o surjan problemas... ${ }^{30}$

Lo que presume una expectativa de afectaciones en los derechos de los empleados, que hace necesario se trabaje en promover una legislación especializada que facilite la transición de la manualidad a la automatización, pero siempre con el respeto a la dignidad del individuo, así como velando por la protección y garantía de sus derechos humanos

En otras palabras,

visualizar aquellos derechos de las personas que van a ser afectados por el uso de la tecnología, los que se podrían empezar a conceptualizar como los derechos de una persona que resiente una disminución o menoscabo en su integridad física o emocional, sus bienes o derechos, producto del uso de la tecnología, sea por interacción laboral, contacto o a distancia en caso de haber solicitado una prestación o servicio digital. ${ }^{31}$

La mencionada resolución del Parlamento Europeo, en el numeral 22 de los principios generales relativos al desarrollo de la robótica y la inteligencia artificial para uso civil:

Pone de relieve que definir normas y posibilitar la interoperabilidad es fundamental para la competencia futura en el ámbito de la inteligencia artificial

30 Parlamento Europeo, Civil Law Rules on Robotics, 16-02-2017, en línea, disponible en: bttp:/ / www.europarl.europa.eu/sides/getDoc.do?pubRef=-//EP//TEXT+TA+P8-TA-2017$0051+0+D O C+X M L+V 0 / / E N($ fecha de consulta: 11 de febrero de 2020).

31 Aguilera Durán, Jesús, "Derecho al trabajo, automatización laboral y derechos de afectación por el uso de tecnología", Revista Latinoamericana de Derecho Social, núm. 29, julio-diciembre de 2018, p. 20. 
y las tecnologías robóticas; pide a la Comisión que continúe trabajando por la armonización internacional de las normas técnicas, en particular junto con los organismos europeos de normalización y la Organización Internacional de Normalización, a fin de fomentar la innovación, evitar la fragmentación del mercado interior y garantizar un elevado nivel de seguridad de los productos y protección de los consumidores, también, en su caso, mediante normas mínimas de seguridad adecuadas para el entorno de trabajo... ${ }^{32}$

Como se puede deducir, hay serias preocupaciones por la interacción entre los robots y los humanos, porque se pueden presentar situaciones en las que una falla mecánica produzca daños, que en un primer plano pudieran ser materiales, pero incluso llegar a perjudicar a las personas a su alrededor.

Por ello, se pretende efectuar cambios legales.

En la propuesta presentada, se aboga por crear una personalidad jurídica específica para los robots, de modo que al menos los robots autónomos más complejos puedan ser considerados personas electrónicas con derechos y obligaciones específicos, incluida la obligación de reparar los daños que puedan causar. ${ }^{33}$

Lo anterior sería una excelente medida de protección para los trabajadores, ya que, si bien es importante la automatización, no se deben dejar de lado sus derechos fundamentales.

Además, se pueden tomar otras medidas, en una primera etapa se debe considerar:

Gravar las nuevas tecnologías, por ejemplo, mediante la introducción de un impuesto a los robots que remplacen el trabajo humano, podría reducir el ritmo de la automatización. Asimismo, un "impuesto a los robots" asimilaría los costos sociales del desplazamiento, aunque queda por saber si esto podría generar incentivos negativos. ${ }^{34}$

32 Parlamento Europeo, op. cit.

33 Mercader Uguina, Jesús R., "El impacto de la robótica y el futuro del trabajo", Revista de la Facultad de Derecho de México, t. LXVII, núm. 269, septiembre-diciembre de 2017, pp. 155 y 156, disponible en: bttp:/ / dx.doi.org/10.22201/fder.24488933e.2017.269.62438 (fecha de consulta: 12 de febrero de 2020).

34 OIT, El futuro del trabajo que queremos: un diálogo global, en línea, p. 6, disponible en: https:/ / www.ilo.org/wcmsp5/groups/public/---dgreports/---cabinet/documents/publication/woms_570288.pdf (fecha de consulta: 9 de febrero de 2020). 
Eso podría remediar, en alguna medida, la pérdida del empleo, porque "En una segunda etapa se podría crear un fondo con los ingresos procedentes de este impuesto para financiar la capacitación (o la nueva capacitación) de los trabajadores más afectados por esta transición". 35

De esa forma, se daría prioridad a la formación profesional del ser humano que le permita disminuir sus desventajas respecto de los robots. No obstante, es impostergable la reflexión sobre lo que se debe hacer hoy para planear un mejor mañana en términos del mundo laboral.

En primer lugar, es necesario ampliar el concepto de «trabajo» e incluir el trabajo no remunerado (y, en particular, el cuidado — de terceros-) para que el concepto no se limite al trabajo remunerado. En segundo lugar, sería recomendable considerar la tecnología como un producto social, con lo cual sus aspectos negativos (como la reducción del empleo) podrían ser controlados mediante la agenda social. ${ }^{36}$

En ese orden de ideas, se hace necesario girar el timón hacia una formación integral de los trabajadores, ya sea antes o cuando estén empleados, para adaptarse a los cambios que la tecnología propicia, que incluya la educación sobre sus derechos fundamentales, la capacitación en informática, en tecnologías de la información y la comunicación y en la adición o la actualización de programas de estudio enfocados a nuevos empleos.

Consecuentemente, se estarían sentando las bases para que los derechos de los trabajadores fueran contemplados de forma holística, y no solamente como derechos de un sector de la sociedad, y asumir una actitud consciente para lograr que la regulación de los derechos fundamentales de los trabajadores frente al uso de la inteligencia artificial sea considerada una política de Estado.

"Yo creo que estamos en el punto ideal en el que podemos desarrollar un marco jurídico eficiente y que pueda crecer a la par de la tecnología o por lo menos pueda adecuarse a los avances tecnológicos", señaló el especialista [Jesús Manuel Niebla Zatarain], quien con el apoyo del Conacyt realiza un

\footnotetext{
35 Ibidem, p. 6.

36 Ibidem, pp. 9 y 10.
} 
doctorado en Inteligencia Artificial y Derecho en la Universidad de Edimburgo, Escocia. ${ }^{37}$

Con el propósito de alcanzar ese objetivo, se debe reglamentar en las leyes laborales, 1) el derecho al trabajo desde casa; 2) el derecho del trabajador a la desconexión de los servidores de la empresa; 3) el acceso a un seguro de desempleo ocasionado por la automatización; 4) la creación de institutos y universidades tecnológicas con horarios flexibles para personas en trabajo activo, y 5) la reducción de la semana laboral.

\section{CONCLUSIONES}

on el uso de la inteligencia artificial en los procesos de producción, no tan sólo se pierden trabajos que desempeñaban los humanos, sino que se propicia el enriquecimiento de los patrones y la vulneración de los derechos laborales de las personas, lo que obliga a asumir una actitud más acorde con la realidad.

También se pone de manifiesto que la capacitación constante en el uso y acceso a la tecnología ya no es una opción, sino una necesidad de prepararse para que en el futuro se pueda competir por empleos que requieren habilidades, destrezas y conocimientos informáticos.

Sin duda, el establecer una regulación sobre los robots otorgaría mecanismos de defensa a los trabajadores para hacer valer sus derechos a la hora de ser desplazados de su empleo o ser perjudicados por alguno de ellos.

Además, se considera pertinente que se proyecte una legislación que incluya la robot's liability o responsabilidad de los robots en su interacción con los trabajadores, que incluya disposiciones de carácter laboral y civil para mantener protegidos los derechos fundamentales de las personas a su alrededor.

La reconfiguración de los derechos fundamentales de los trabajadores en las nuevas formas de trabajo debe incluir el reconocimiento de condiciones económicas y de protección social que incluyan capacitación laboral, seguro de desempleo, acceso a educación especializada, el fortalecimiento de los sindicatos en su ámbito interno de la empresa y todos aquellos derechos que reduzcan la inequidad de condiciones propiciada por el uso de la robótica y la inteligencia artificial en el ámbito laboral.

37 Sánchez, Verenice, "Inteligencia artificial y las leyes del futuro", Conacyt prensa, en línea, disponible en: http:/ / conacytprensa.mx/index.php/sociedad/personajes/2293-inteligencia-artificial-y-lasleyes-del-futuro (fecha de consulta: 13 de febrero de 2020). 


\section{FUENTES DE INFORMACIÓN}

Aguilera Durán, Jesús, "Derecho al trabajo, automatización laboral y derechos de afectación por el uso de tecnología", Revista Latinoamericana de Derecho Social, núm. 29, julio-diciembre de 2018.

Aguilera Durán, Jesús, "Derecho de asociación de los trabajadores de confianza del municipio en el Estado de Morelos", en CABRERA DIRCIO, Julio et al., Las reformas constitucionales y su impacto en el municipio, México, Editorial Fontamara, 2015.

BONET PÉREZ, Jordi, "Disrupción tecnológica y trabajo, ¿disrupción también en el ámbito de las relaciones laborales?", Revista Mientras tanto.e, en línea, núm. 169, junio de 2018, disponible en: http:/ / wmw.mientrastanto.org/ boletin-169/notas/disrupcion-tecnologica-y-trabajo-disrupcion-tambien-en-el-ambitode-las-relaciones.

CARrasco FERnÁnDEZ, Felipe Miguel, "Rescisión laboral por uso de medios electrónicos en la empresa”, en IBARRA SÁNCHEZ, Ernesto y ROMERO FLORES, Rodolfo, Jurismática. El derecho y las nuevas tecnologías, UANL, 2010.

DACHS, Bernhard, The Impact of New Technologies on the Labour Market and the Social Economy, European Parliament, 2018.

GIDDENS, Anthony, As consequencias da modernidade, Sao Paulo, Editora UNESP, 1991.

HE, David y VANESSA, Guo, "4 ways AI [Artificial Intelligence] will Impact the Financial Job Market", World Economic Forum, disponible en: https:/ / www.weforum.org/agenda/2018/09/4-ways-ai-artificial-intelligence-impact-financial-job-market/.

IFR International Federation of Robotics, Global industrial robot sales doubled over the past five years, disponible en: https: / ifr.org/ifr-press-releases/news/globalindustrial-robot-sales-doubled-over-the-past-five-years.

ISO TOOLS, “¿Qué es una norma ISO?”, disponible en: https:/ / wmw.isotools. org/normas/.

MCKINZEY GLOBAL INSTITUTE, Un futuro que funciona: automatización, empleo y productividad, Resumen ejecutivo, 2017, disponible en: https://www. mckinsey.com/ / media/ mckinsey/featured\%20insights/digital\%20disruption/ harnessing $\% 20$ automation $\% 20$ for $\% 20$ a\%20future $\% 20$ that $\% 20$ works / a-future-thatwork-executive-summary-spanish-mgi-march-24-2017.ashx'. 
Mercader Uguina, Jesús R., "El impacto de la robótica y el futuro del trabajo", Revista de la Facultad de Derecho de México, t. LXVII, núm. 269, septiembre-diciembre de 2017, disponible en: http:/ / dx.doi.org/10.22201/ fder.24488933e.2017.269.62438.

OACNUDH (Oficina del Alto Comisionado de Naciones Unidas para los Derechos Humanos), disponible en: http://acnudh.org/wpcontent/ uploads/2010/12/carta-PF-PIDESC-FINAL.pdf.

OIT, El futuro del trabajo que queremos: un diálogo global, disponible en: https:/ / wwn.ilo.org/womsp5/groups/public/---dgreports/---cabinet/documents/publication/ wcms_570288.pdf.

PALMERINI, Erika, "Robótica y derecho: sugerencias, confluencias, evoluciones en el marco de una investigación europea", Revista de Derecho Privado, núm. 32, enero-junio de 2017, disponible en: https:/ / revistas.uexternado.edu. co/index.php/derpri/article/view/5021/6009.

Parlamento Europeo, Civil Law Rules on Robotics, disponible en: http:/ / www.europarl.europa.eu/sides/getDoc.do?pubRef=-//EP//TEXT+TA+P8TA-2017-0051+0+DOC+XML+V0//EN.

PASCUAL, Álvaro David, Inteligencia artificial: un panorama de algunos de sus desafios éticos y jurídicos, Universitat de Girona, disponible en: https:/ / dugidoc.udg.edu/ bitstream/ handle/10256/14950/alvaro-pascual.pdf? sequence $=1$.

Repositorio Institucional de la Fundación Universitaria San Pablo CEU, Informe ADDECO sobre el futuro del trabajo en España 2016, disponible en: https:/ / www.ceu.es/joblab/documentacion/informeAdecco.pdf.

SÁNCHEZ, Verenice, "Inteligencia artificial y las leyes del futuro", Conacytprensa, disponible en: http:/ / conacytprensa.mx/index.php/sociedad/personajes/2293inteligencia-artificial-y-las-leyes-del-futuro.

U. S. White House, Economic Report of the President 2016, disponible en: https:/ / obamawhitehouse.archives.gov/sites/default/files/docs/ERP_2016_Chapter_5.pdf

VILLAMIL, Jenaro, "El Senado ratifica el Convenio 98 sobre derechos sindicales y contra contratos de protección patronal", Revista Proceso, núm. 2190, 20 de octubre 2018, disponible en: https:// wmw.proceso.com.mx/551845/ el-senado-ratifica-el-convenio-98-sobre-derechos-sindicales-y-contra-contratos-de-proteccion-patronal.

ZAFrA, Juan Manuel, "Más humano y digital", TELOS Revista de Pensamiento, Sociedad y Tecnología, núm. 108, enero-junio de 2018. 